\title{
Texte littéraire et interculturalité : enjeux didactiques
}

Errafiq Brahim, Université Chouaib doukali jadida, Maroc

Il va de soi que la mondialisation qui touche les échanges économiques et culturels semble aller dans le sens d'une uniformisation des différences. Les revendications identitaires liées à la culture sont conséquentes à cette globalisation. La différence et la diversité culturelles devenant le paradigme central dans les politiques de gouvernance et du management, doivent l'être aussi dans la gestion pédagogique et didactique au sein des institutions éducatives. En effet, la mission de l'espace éducatif ne se limite plus à la maitrise des langues mais la dépasse pour porter son intérêt sur le traitement des cultures afin d'assurer une éducation interculturelle aux apprenants. L'espace universitaire marocain ne saurait déroger à une telle mission, car l'université est appelée à apporter des réponses aux questionnements autour de la culture, de l'identité, de l'altérité et des représentations via une didactique interculturelle. Dans ce sens, il nous semble que l'enseignement du français par le texte littéraire dans les départements de langue et littérature françaises importe du moment que c'est par le texte littéraire que transitent les représentations et les transferts interculturels. En tant que production de l'imaginaire, il représente un genre inépuisable pour la rencontre de l'Autre. La littérature permet d'étudier l'être humain dans sa complexité et sa variabilité. Nous arrivons aux questions problématiques suivantes : l'enseignant universitaire souscrit-il à une didactique interculturelle qui initie une réflexion constructive de l'étudiant sur l'identité et l'altérité et promeut un apprentissage de la décentration et de dépassement du soi ? Quels enjeux didactiques affectent «l'enseignabilité » de la compétence interculturelle par le truchement du texte littéraire? C'est à ces questions que nous tentons d'apporter des éléments de réponse en prenant appui sur les résultats d'une enquête que nous avons menée auprès des professeurs de littérature française exerçant dans les départements de langue et littérature françaises au sein des facultés des lettres marocaines. Nous présentons dans cet article un descriptif de l'enquête suivi de l'exposition des résultats et de leur interprétation.

\section{Cadre et descriptif de la recherche}

Cette étude fait l'objet de notre thèse de doctorat intitulée «texte littéraire et interculturalité : enjeux didactiques ». Cette recherche, qui s'inscrit dans le cadre de la didactique des langues-cultures, ambitionne le diagnostic des enjeux qui sous-tendent la mise en place d'une didactique interculturelle appliquée au texte littéraire dans l'espace 
universitaire marocain, notamment dans les départements de langue et littérature françaises. Nous sommes parti des postulats de base suivants :

-Inscrire le texte littéraire dans une optique interculturelle contribuerait à la construction identitaire de l'apprenant et à son ouverture sur l'altérité.

-Dans les pratiques enseignantes, le texte littéraire demeure beaucoup plus un espace d'apprentissage techniciste et fonctionnel de la langue et de la communication qu'un espace d'étude des représentations interculturelles, de rencontre de l'altérité et d'apprentissage de la décentration.

-Les enseignants sont peu ou pas du tout outillés pour promouvoir une didactique interculturelle du texte littéraire.

-En l'absence de professeurs-médiateurs interculturels, les chances de développer la compétence interculturelle de l'étudiant s'amenuisent.

Pour répondre à notre problématique et vérifier nos hypothèses, nous avons mené une enquête par questionnaire auprès de 53 professeurs de littérature exerçant dans le supérieur marocain. Cette recherche quantitative a été alimentée par des observations de classe. Elle comporte les axes suivants :

\section{1-Identification des sujets :}

Cette catégorie ne vise pas à nommer la personne. Il s'agit surtout de relever certaines caractéristiques du sujet en mesure de le situer en tant qu'enseignant de la littérature au sein de la population ciblée par l'enquête.

\section{2-Culture française et représentations des enseignants :}

Cet axe vise à identifier les représentations des interrogés sur la francité : la France, la culture française et les Français.

\section{3-Formation à l'interculturel :}

Cette catégorie se propose de déterminer si les enseignants ont été formés à la didactique de l'interculturel et si la formation en question leur a permis d'enseigner avec aisance ou difficilement selon une perspective interculturelle.

\section{4-Planification de l'enseignement interculturel :}

Cette catégorie vise à vérifier si :

- le contenu enseigné découle d'un cadre de référence, plus précisément d'un référentiel de compétences (inter)culturelles ;

- les interrogés diagnostiquent les besoins (inter)culturels des étudiants ;

- pour chaque séance, séquence et module les interrogés définissent des objectifs (inter)culturels. 


\section{5-Texte littéraire : compétence interculturelle et transposition didactique}

Il s'agit pour le sujet répondant de déterminer quelle pédagogie il applique en classe, quel est son style d'enseignement, quelles techniques d'animation il adopte pour favoriser un enseignement interactif. Les enseignants sont aussi interrogés pour examiner s'ils associent l'enseignement de la langue et de la culture françaises, sur quelle composante ils tablent le plus souvent dans leurs pratiques et s'ils recourent à la langue-culture des étudiants. L'enquêteur s'interroge sur le type d'approche mis en vigueur par les interrogés, sur les critères qui président au choix du texte littéraire, sur le contenu interculturel du texte en question et sur les types de documents utilisés pour nourrir les supports littéraires. Les enquêtés sont appelés aussi à indiquer les objectifs inhérents à la compétence interculturelle et aux autres activités auxquelles ils recourent pour développer la compétence en question chez l'étudiant. L'interrogé indiquera aussi quel geste professionnel il met en œuvre pour traiter les implicites culturels, les stéréotypes et les chocs culturels enclenchés par le document littéraire étudié. Il s'agit en dernier lieu de vérifier si la compétence interculturelle est évaluée et selon quelles modalités.

\section{6- Texte littéraire et perspective interculturelle : écueils et alternatives}

Cette dernière catégorie a pour objectif de vérifier si les interrogés réussissent à inscrire leur enseignement dans une perspective interculturelle. Les répondants citeront aussi les principaux obstacles qui entravent la perspective en question et suggèreront des solutions pour promouvoir une didactique de l'interculturel par le truchement du support littéraire.

\section{Présentation des résultats}

Le dépouillement du questionnaire montre que :

Les enquêtés, quoique différents par leurs grades, présentent une certaine homogénéité : ils sont tous professeurs marocains de littérature française titulaires d'un doctorat en littérature française et ayant exercé plus de quinze ans ;

$-90 \%$ des interrogés ont des représentations positives de la France, des Français et de la langue et de la culture françaises ;

-Les enseignants interrogés n'ont bénéficié d'aucune formation initiale ou continue portant sur l'interculturel ;

-les interrogés ne se réfèrent à aucun référentiel de compétences (inter)culturelles, ne diagnostiquent pas les besoins interculturels des étudiants et rares (12\%) sont ceux qui définissent un objectif interculturel pour leurs cours ; 
-les sujets de l'enquête (95\%) font appel à l'approche communicative, les autres (5\%) assurent un enseignement transmissif et directif ;

$-40 \%$ des répondants associent l'enseignement de la dimension culturelle à la dimension linguistique de la langue cible et pour la quasi-totalité des enseignants (95\%) le linguistique est prioritaire ;

-20\% des enquêtés convoquent la langue-culture de l'apprenant ;

-concernant le type d'approche interculturelle appliquée au texte littéraire, tous les enquêtés avancent qu'ils adoptent l'approche anthropologique du texte littéraire comme moyen, qu'ils choisissent les textes littéraires en fonction d'objectifs stylistiques et n'alimentent pas ces textes par d'autres documents en mesure d'assurer l'interculturalité ;

-pour $60 \%$ des interrogés, la compétence interculturelle favorise la réussite scolaire, $15 \%$ des répondants pensent qu'elle permet la reconnaissance de la diversité culturelle et $25 \%$ des enseignants avancent qu'elle favorise l'enrichissement culturel ;

-aucun interrogé n'introduit d'autres activités pour développer la compétence interculturelle des étudiants ;

-face aux stéréotypes et aux chocs culturels enclenchés par le texte littéraire, les enseignants sont unanimes à dire qu'ils laissent les étudiants en discuter et adoptent une attitude objective ;

-peu d'enseignants (10\%) évaluent la compétence interculturelle de leurs étudiants ;

-tous les sujets interrogés peinent à assurer un enseignement à dimension interculturelle ;

-les obstacles récurrents qui entravent la mise en place de la didactique interculturelle sont :

Manque de formation à l'interculturel

Niveau linguistique insatisfaisant des étudiants

Manque de référentiel de compétences interculturelles

Effectif élevé des étudiants (classes pléthoriques)

Manque de matériel didactique adéquat

-la seule alternative proposée par tous les professeurs est la nécessité pour le ministère de l'enseignement supérieur de concevoir des formations initiales et continues portant sur la didactique de l'interculturel et de promouvoir des échanges interculturels entre les étudiants et les natifs de la langue. 


\section{Interprétation et discussion des résultats}

Les données recueillies s'interprètent à plusieurs niveaux .Nous nous limitons à ce stade à ceux qui sont en rapport avec le traitement interculturel du texte littéraire dans le contexte universitaire marocain. Nous soulignons que nous comprenons la notion d'interculturel telle qu'elle a été définie par le Conseil de l'Europe (1986):

L'emploi du mot interculturel implique nécessairement, si on attribue au préfixe inter sa pleine signification, interaction, échange, élimination des barrières, réciprocité et véritable solidarité. Si on reconnaît toute sa valeur au terme « culture », cela implique reconnaissance des valeurs, des modes de vie et des représentations symboliques auxquels les êtres humains, tant les individus que les sociétés, se réfèrent dans les relations avec les autres et dans la conception du monde. (L'interculturalisme)

La légitimation de l'introduction de la perspective interculturelle dans l'enseignement des langues s'avère motivée et a dorénavant droit de cité dans le milieu éducatif, d'autant plus qu'actuellement l'école ne peut plus ignorer les enjeux identitaires qui font surface dans la sphère publique. A ce propos, Geneviève Vinsonneau et al avancent :

Aujourd'hui dans les sociétés démocratiques modernes on se sent concerné par la montée du communautarisme, l'affirmation, parfois tapageuse et plus ou moins dramatique sur la scène politique, des appartenances des uns et des autres. Les revendications identitaires, autrefois reléguées dans l'espace privé, s'expriment désormais sur la scène publique, prétendant à la légitimé dans des espaces jusquelà protégés de ces phénomènes. C'est ce qui se passe à l'école. L'ethnicisation des conduites et des discours s'immisce dans ses murs, ébranlant son principe de laïcité et menaçant sa vocation à l'éducation et à l'universel. (11)

Dans les contextes sociaux traversés par de telles turbulences, un consensus s'établit chez les agents des systèmes scolaires soucieux de développer une éducation interculturelle. Ils s'accordent en l'occurrence pour proposer d'inculquer aux élèves des valeurs démocratiques, pour les informer sur la question du pluralisme ethnoculturel et pour développer leurs capacités à vivre ensemble : dans l'harmonie et la perspective d'une société d'égalité de droit et d'équité.

Il est à souligner que le texte littéraire constitue le médium où se déploie l'interculturalité par excellence. L'œuvre littéraire peut donc constituer une voie d'accès à des différents codes sociaux et à des visions du monde dans la mesure où elle représente une mosaïque assez expressive du désir de soi et de l'autre. A ce propos, Martine AbdallahPretceille affirme que « le texte littéraire, production de l'imaginaire, représente un genre inépuisable pour l'exercice artificiel de la rencontre avec l'Autre ; rencontre par procuration certes, mais rencontre tout de même » $(1999,2)$. La littérature permet une confrontation avec l'altérité : 
L'autre en tant qu'autre, c'est-à-dire comme moi, un sujet (responsable et absolument singulier, incomparable), il est à la fois différent de moi et identique à moi en dignité. L'altérité est le concept qui recouvre l'ensemble des autres, considérés eux aussi comme des ego (alter ego) et dont je suis moi aussi l'alter ego, avec droit et devoirs. Pour être moi, j'ai besoin que les autres (l'altérité) existent. Tout sujet suppose une intersubjectivité et en même temps éprouve toujours la tentation de réduire l'autre à un objet, grand danger contre lequel il faut sans cesse lutter en soi même pour les relations humaines. Cuq (17)

L'altérité se définit globalement par rapport à l'identité définie par Prétceille comme « une étiquette, une réalité observable et un processus dynamique enclenché par un individu ou un groupe en réponse à une situation donnée. C'est ainsi que selon les circonstances, les stratégies, l'accent sera mis sur la langue, le territoire, la religion » $(1986,64)$.

Le texte littéraire referme souvent une représentation du monde, des valeurs partagées d'une culture à une autre, encore faut-il savoir comment mettre en exergue ce commun héritage par une pratique interculturelle du texte littéraire.

.Qu'en est-il de l'interculturalité dans l'université marocaine?

D'après les réponses des enquêtés, on note la non prise au sérieux de l'interculturel par les praticiens, cela s'atteste à plusieurs niveaux :

\section{Au niveau de la formation des enseignants :}

La lecture des réponses des interrogés laisse entendre que les professeurs ne sont pas formés à la didactique interculturelle, que cela soit dans le cadre d'une formation initiale ou dans le cadre d'une session de formation continue. Et c'est l'une des raisons qui expliquent pourquoi l'interculturalité n'est pas opératoire à l'université. Notons à ce propos que, faute de formation à l'interculturel, certains professeurs s'auto-forment en consultant, par exemple, les publications du Conseil de l'Europe (le cadre européen de référence pour les langues) qui donnent aux enseignants des pistes pédagogiques pour inscrire la dimension interculturelle dans l'enseignement-apprentissage des langues vivantes. Elles proposent par ailleurs un kit pédagogique avec des idées, des ressources, des méthodes et des activités pour l'éducation interculturelle avec des adultes et des jeunes.

\section{Au niveau de la planification des apprentissages :}

Il n'existe aucun référentiel de compétences (inter)culturelles auquel peuvent se référer les enseignants pour planifier les contenus à enseigner. Un tel référentiel contribuerait à orienter l'enseignant et à lui indiquer le socle de compétences interculturelles que doit maitriser l'étudiant au terme d'un module. Ce manque donne au professeur la liberté de prendre l'initiative mais donne lieu parfois à l'improvisation et à des pratiques enseignantes irréfléchies. 
Une planification rationnelle des apprentissages suppose un diagnostic des besoins. Or, les enseignants ne se soucient guère d'identifier les besoins de leurs étudiants (qu'ils soient linguistiques ou culturels) et les représentations des apprenants sur la culture-cible. Notons à ce propos que la notion de représentation est en général prioritairement traitée par les sciences sociales. Emile Durkeim a fait émerger ce concept pour expliquer qu'entre les divers groupes sociaux circulent des images autrui. D'un point de vue étymologique, « représenter », du latin « repraesentare » signifiant « rendre présent », indique bien qu'il y a une tentative de faire exister au travers du discours une réalité (du moins, le sujet la perçoit comme telle) extra-linguistique.

Les représentations sociales (Moscovici ; Abric) qui ont cours au sein d'une communauté affectent aussi les images de la langue parlée par ses membres. Ces images liées à chaque langue en particulier n'ont aucune base scientifique (Jacquart) : elles configurent une série d'attitudes qui sont souvent au centre des motivations qui déterminent le choix d'une langue comme objet d'étude et le processus d'enseignementapprentissage.

Le discours qu'une société tient sur une langue, dépend de nombreux critères d'appréciation («économiques », « sociaux », « épistémiques », « affectifs », etc.) autant de la langue en question que de ses locuteurs ou même de l'espace physique où elle est parlée. Le futur apprenant d'une langue est conditionné par tous ces critères; sur le marché linguistique, sera plus cotée la langue dont l'image répond le mieux à ces questions (et à d'autres) : est-ce une langue qui donne accès à un travail ? Ses locuteurs ont-ils un niveau social ou un pouvoir économique attractif? Quelle est la richesse culturelle ou la valeur esthétique de cette langue ? Quelles ont été les relations historiques avec le(s) pays où elle est parlée ? Ou même d'autres critères encore plus subjectifs : estce une langue facile ou difficile? Quelles sont les relations personnelles du sujet avec cette langue ou avec le(s) pays où elle est parlée? Est-ce la langue d'un pays «exotique »?

Il est donc intéressant dans une perspective interculturelle que le professeur fasse émerger ces représentations pour les faire évoluer.

Concernant toujours la planification des apprentissages, nous déduisons des résultats de l'enquête que les enquêtés ne définissent préalablement dans le cadre de l'étape prépédagogique des objectifs interculturels ni pour la séance, ni pour la séquence et ni pour le module. Les axes linguistiques, thématiques et stylistiques représentent le domaine de prédilection des enseignants. 


\section{Au niveau de la gestion des apprentissages :}

Les enseignants recourent généralement à l'approche communicative qui rime bien avec un enseignement interculturel qui exige davantage de souplesse de la part de l'enseignant puisqu'il s'agit dans l'optique interculturelle d'atteindre des objectifs inhérents au savoir-être tels que la tolérance, l'ouverture sur l'autre, le relativisme culturel etc.

Il ressort aussi des résultats recueillis que les interrogés se considèrent transmetteurs de savoir et pas du tout médiateurs interculturels. Ceci va à l'encontre de l'approche interculturelle. Selon Jean Cuq, la médiation

désigne généralement une relation qui s'opère entre des personnes sous le guidage de tiers spécialistes (des médiateurs). Plus largement, on peut dire que la médiation est présente d'emblée dans le langage dès lors qu'on admet que les mots ne ont pas les choses, même si ces mots veulent désigner, représenter ces choses. La médiation peut donc influencer la relation de l'homme à la réalité du monde et, en ce sens, elle est centrale dans l'analyse des représentations sociales et dans l'approche interculturelle. (163)

La médiation interculturelle consiste à établir des liens de sociabilité entre des gens issus de cultures différentes. Elle a pour but d'identifier des conflits relevant de l'interaction humaine et de proposer des stratégies pour résoudre ces problèmes. Les conflits résultent non seulement des différentes identités nationales, mais également des divergences au niveau des traditions et des cultures. La base d'une communication interculturelle réussie est la compétence émotionnelle. Il va sans dire qu'un enseignement interculturel suppose un maitre interculturel qui sait se décentrer de ses cribles culturels et favoriser la négociation des points de vue en classe en recourant à des techniques d'animation spécifiques telles le brainstorming, les débats, les exposés, etc. ; ce qui n'est pas le cas pour les interrogés, qui analysent le texte littéraire suivant une modalité magistrale qui fait peu de place à l'interaction.

Les réponses des enseignants attestent également que rares sont ceux qui associent l'enseignement de la langue à la culture. L. Porcher montre que, dans le principe épistémologique, «langue et culture sont inséparables »(Civilisation, 33-44). Il met en avant ces arguments :

-La culture dans la langue : une langue n'est pas seulement un système formel, un ensemble de réalités abstraites logiquement structurées. Elle est aussi (d'abord, surtout) une pratique sociale, c'est-à-dire non indépendante de ceux qui s'en servent. La linguistique ellemême, comme science, a établi ce constat, notamment au travers des linguistiques 
énonciatives, des pragmatiques, des sociolinguistiques. Il y a, en somme, pour reprendre une formulation que certains linguistes récuseraient probablement, du culturel dans le linguistique. Dans ces conditions, en enseignant la langue on enseignerait nécessairement aussi un certain nombre de valeurs et de fonctionnements et culturels, même sans le vouloir. Donc, didactiquement, il vaut mieux le savoir et même l'expliciter. On peut considérer que, dans la didactique d'aujourd'hui, en tout cas, un très large consensus existe sur cette présence du culturel dans le linguistique

-La culture dans la communication : Les approches communicatives en didactique du français langue étrangère reposent, entre autres principes, sur l'idée que la communication, comme réalité sociale et comme pratique ne se réduit pas à la communication linguistique. Il y a du non linguistique dans la communication, et, dans ces conditions, il ne suffit pas d'enseigner du linguistique pour que les apprenants apprennent à communiquer. Construire chez l'apprenant une compétence de communication suppose qu'on lui fasse maitriser, dans la langue étrangère, un certain nombre de phénomènes extralinguistiques spécifiques de la société dans laquelle cette étrangère est langue native (ou maternelle). Ces phénomènes extralinguistiques sont manifestement de nature culturelle : gestualité, mimique, expression corporelle et psychologie posturale, font partie de cet ensemble à travers la kinésique, la proxémique, la topologie sociale : «Toute langue véhicule avec elle une culture dont elle est à la fois la productrice et le produit »(Porcher, Français, 53)

La relation langue-culture, bien étroite, sinon les deux faces d'une même pièce, est constitutive du canal nécessaire à la transposition didactique. Or, comment peut-on enseigner une langue, de surcroit étrangère, sans la construire sur la base d'un socle, en l'occurrence la variable «culture », car une langue déculturée est une langue morte.

On note également qu'à travers les réponses des interrogés, la plupart des enseignants ne convoquent pas la langue-culture des apprenants. Or, didactiquement parlant, si on s'inscrit dans une optique interculturelle, il est nécessaire de comparer des faits interculturels en convoquant simultanément la culture-source et la culture-cible ; cela sensibilise l'étudiant au relativisme culturel, car toutes les cultures sont égales en dignité.

La compétence interculturelle est aussi un des items sur lequel se sont prononcés les interrogés. Elle peut être conçue comme étant la capacité du locuteur-auditeur à saisir, à comprendre, à expliquer et à exploiter positivement les données pluriculturelles ou multiculturelles dans une situation de communication donnée. Une telle définition, pense Abdallah-Pretceille, n'implique pas une simple connaissance descriptive des cultures ou une simple connaissance des faits de civilisation, «mais une maîtrise de la situation de 
communication dans sa globalité, dans sa complexité et dans ses multiples dimensions (linguistique, sociologique, psychologique...et culturelle) » $(1996,29)$. On voit mal comment les enseignants développent cette compétence en l'absence d'une approche particulière (anthropologique, pragmatique, sémiologique, lexicologique, ethnographique, par l'implicite, par les stéréotypes, etc.) et à défaut d'un traitement didactique des stéréotypes véhiculés par les textes littéraires étudiés et des chocs culturels éventuels qu'enclenchent certains textes. Les répondants affirment que, face aux stéréotypes et aux chocs culturels, ils se tiennent à l'écart et laissent les étudiants discuter loin de toute médiation interculturelle. En principe, si des stéréotypes émergent, le professeur doit les déconstruire et relativiser leur véracité ; l'enseignant se doit aussi d'analyser les implicites culturels contenus dans le texte et apprendre à l'élève que le texte est d'essence polysémique par le croisement des regards. Quant aux chocs culturels, le professeur habituera l'apprenant à se distancier de ses schèmes culturels pour relativiser son système de valeurs et ce, à travers le processus d'acculturation. On désigne par acculturation l'ensemble des phénomènes qui résultent de ce que les groupes d'individus de cultures différentes entrent en contact, continu et direct, avec les changements qui surviennent dans les patrons culturels originaux de l'un ou des deux groupes. Selon cette définition, l'acculturation doit être distinguée du changement culturel, dont elle n'est que l'un des aspects, et de l'assimilation, qui n'en est que l'une de phases. Face à un choc culturel, l'apprenant passe par ces phases. A une première phase ethnocentrique où interviennent les préjugés, s'ensuit une phase d'ajustement par rapport à cette situation; la troisième phase survient avec la confrontation et le stress. Le problème de l'identité se pose souvent à ce moment. Le quatrième stade est celui de l'ajustement au stress. L'enseignant prenant connaissance de ces phases d'acculturation saura gérer le stress enclenché par les chocs culturels.

Au niveau de l'évaluation, les professeurs affirment unanimement qu'ils n'évaluent pas la compétence interculturelle de leurs étudiants. Ce qui prime à leurs yeux, c'est la compétence linguistique et communicative. S'ils évaluent un aspect interculturel, c'est généralement par le biais des interactions écrites (commentaire composé, dissertation). Ils ont aussi souligné qu'ils peinent à assurer un enseignement interculturel.

\section{Conclusion}

Les résultats de notre recherche font ressortir que l'interculturalité n'a pas encore droit de cité dans le contexte universitaire marocain. Si le texte littéraire constitue une voie d'accès au divers et un espace de rencontre d'autrui, les enseignants s'en servent dans leurs pratiques pour répondre à des objectifs linguistiques et communicatifs. En assurant un enseignement 
techniciste de la langue, les enseignants dérogent à leur mission de médiateurs interculturels sans laquelle on ne peut pas favoriser la construction de l'identité de l'apprenant et son ouverture à l'altérité. L'enquête nous a révélé que, faute d'une approche culturelle ancrée dans les pratiques de classe, il est impossible de mettre en place un enseignement interculturel. Les professeurs sont peu ou pas du tout outillés pour inscrire l'interculturalité dans leurs pratiques. Devant le manque de formation à l'interculturalité, l'inexistence d'un référentiel de compétences, le niveau linguistique insatisfaisant de étudiants, les effectifs pléthoriques, l'insuffisance des moyens didactiques, l'interculturel devient lettre morte, une utopie et, du coup, les résistances des enseignants quant à la mise en place de la didactique interculturelle ne manquent pas de s'accentuer. Tout porte à croire que la culture marocaine se conjugue au pluriel. La diversité est partout perceptible, ce qui fait l'originalité de la culture maghrébine par rapport aux autres pays arabes et africains, mais l'école ne prend pas en charge cette diversité. Nous pensons qu'une bonne éducation doit donc, dans la matrice de la modernité, aider les élèves à dépasser les frontières de leur culture particulière pour composer avec d'autres cultures et cela ne peut s'objectiver qu'à travers la conception d'une didactique interculturelle ou par «l'intercompréhension», nouvelle approche pour l'enseignement des langues que prône Sandrine Caddéo. Eu égard aux résultats de notre recherche, nous projetons actuellement, dans le cadre d'une action de recherche, de concevoir les bases d'une didactique interculturelle adaptée au contexte marocain où les objectifs, les besoins culturels, les progressions et les modalités d'évaluation soient clairement énoncés et définis.

\section{Bibliographie}

Abdallah-Pretceille Martine. L'éducation interculturelle. Paris : PUF, 1999.

---. Éducation et communication interculturelle. Paris : PUF, 1996.

---. Vers une pédagogie interculturelle. Paris : P de la Sorbonne, 1986.

Bourdieu, Pierre. La distinction, critique sociale et jugement. Paris : Minuit, 1984.

Caddeo, Sandrine. L'intercompréhension: une autre approche pour l'enseignement des langues. Paris : Hachette, 2013.

Cuq, Jean. Dictionnaire de didactique du français langue étrangère et seconde. Paris : Clé International, 2003.

--- et GRUCA. Cours de didactique du français langue étrangère et seconde. Grenoble : PU de Grenoble, 2005. 
Jacquart, A. «L'amour des langues. Représentations linguistiques et motivations du choix d'une langue étrangère ». Cahiers de l'ASDIFLE (1997) : 75-186.

L'interculturalisme : de l'idée à la pratique didactique et de la pratique à la théorie. Strasbourg : Conseil de l'Europe, 1986.

Porcher, L. La civilisation. Paris : Clé international, 1986.

---. Le français langue étrangère, émergence et enseignement d'une discipline. Paris: Hachette, 1995.

Vinsonneau, Geneviève et al. L'interculturel : principes et réalités à l'école. Paris : Sides, 2004.

Zakhartchouk, J-M. L'enseignant, un passeur culturel. Paris, ESF. 1999.

Zarate, Geneviève. Représentation de l'étranger en didactique des langues. Paris : CrédifDidier, 1993.

---. Enseigner une culture étrangère. Paris : Hachette, 1986. 